\title{
Cell Adhesion Process
}

National Cancer Institute

\section{Source}

National Cancer Institute. Cell Adhesion Process. NCI Thesaurus. Code C41514.

A cellular process initiated by cell adhesion molecules which results in the attachment of a cell to either another cell or to an underlying substrate such as the extracellular matrix. 